\title{
Article \\ Lowering the Intraocular Pressure in Rats and Rabbits by Cordyceps cicadae Extract and Its Active Compounds
}

\author{
Li-Ya Lee ${ }^{1,2}$, Jui-Hsia Hsu ${ }^{2}$, Hsin-I Fu ${ }^{2}$, Chin-Chu Chen ${ }^{2,3,4,5, *}$ and Kwong-Chung Tung ${ }^{1, *}$ \\ 1 Department of Veterinary Medicine, National Chung Hsing University, Taichung 402204, Taiwan; \\ ly.lee@grapeking.com.tw \\ 2 Biotech Research Institute, Grape King Bio Ltd., Taoyuan 325002, Taiwan; \\ juihsia.hsu@grapeking.com.tw (J.-H.H.); hsini.fu@grapeking.com.tw (H.-I.F.) \\ 3 Institute of Food Science and Technology, National Taiwan University, Taipei 104336, Taiwan \\ 4 Department of Food Science, Nutrition and Nutraceutical Biotechnology, Shih Chien University, \\ Taipei 104336, Taiwan \\ 5 Department of Bioscience Technology, Chung Yuan Christian University, Taoyuan 320314, Taiwan \\ * Correspondence: gkbioeng@grapeking.com.tw (C.-C.C.); kctung1@dragon.nchu.edu.tw (K.-C.T.)
}

check for updates

Citation: Lee, L.-Y.; Hsu, J.-H.; Fu, H.-I.; Chen, C.-C.; Tung, K.-C. Lowering the Intraocular Pressure in Rats and Rabbits by Cordyceps cicadae Extract and Its Active Compounds. Molecules 2022, 27, 707. https:// doi.org/10.3390/molecules27030707

Academic Editors: Luca Santi, Laura Dugo and Francesca Rigano

Received: 5 December 2021

Accepted: 13 January 2022

Published: 21 January 2022

Publisher's Note: MDPI stays neutral with regard to jurisdictional claims in published maps and institutional affiliations.

Copyright: (C) 2022 by the authors. Licensee MDPI, Basel, Switzerland. This article is an open access article distributed under the terms and conditions of the Creative Commons Attribution (CC BY) license (https:// creativecommons.org/licenses/by/ $4.0 /)$.

\begin{abstract}
Cordyceps cicadae (CC), an entomogenous fungus that has been reported to have therapeutic glaucoma, is a major cause of blindness worldwide and is characterized by progressive retinal ganglion cell (RGC) death, mostly due to elevated intraocular pressure (IOP). Here, an ethanolic extract of $C$. cicadae mycelium (CCME), a traditional medicinal mushroom, was studied for its potential in lowering IOP in rat and rabbit models. Data showed that CCME could significantly $(60.5 \%)$ reduce the IOP induced by microbead occlusion after 56 days of oral administration. The apoptosis of retinal ganglion cells (RGCs) in rats decreased by $77.2 \%$. CCME was also shown to lower the IOP of normal and dextrose-infusion-induced rabbits within $60 \mathrm{~min}$ after oral feeding. There were dose effects, and the effect was repeatable. The active ingredient, N6-(2-hydroxyethyl)-adenosine (HEA), was also shown to alleviate $29.6 \%$ IOP at $0.2 \mathrm{mg} / \mathrm{kg}$ body weight in this rabbit model. CCME was confirmed with only minor inhibition in the phosphorylated myosin light chain 2 (pMLC2) pathway.
\end{abstract}

Keywords: Cordyceps cicadae; intraocular pressure (IOP); ganglion cells; N6-(2-hydroxyethyl)-adenosine (HEA)

\section{Introduction}

C. cicada is an entomopathogenic fungus that produces fruit on the head of the host (Cicada flammata) and then forms a fruiting body on the surface of the insect. The hybrid of fruiting body and insect has been used as traditional Chinese medicine for 1600 years. It possesses many medical effects similar to Hirsutella sinensis such as antioxidation [1,2], anti-inflammation [3,4], lowering blood sugar [5], and renal protection [6,7]. Zheng's findings indicated that HEA, an active compound from C. cicadae, has a beneficial effect on UUO-induced tubulointerstitial fibrosis by suppressing inflammation and renal fibroblast activation. Inhibiting renal fibrosis of $C$. cicadae in vivo may be through the TGF- $\beta 1 / C T G F$ pathway [8]. Treatment with HEA (20 and $40 \mathrm{mg} / \mathrm{kg}$ ) for six weeks reduced blood glucose in alloxan-induced diabetic rats [9]. The safety of C. cicadae mycelium has been evaluated in rat, rabbit, pig, and mouse models, as well as humans [10-19].

Recent studies on novel applications of $C$. cicadae have focused on eye health. C. cicadae-fermented mycelia extract in a benzalkonium chloride (BAC)-induced dry eye model has been evaluated in mice. $C$. cicadae extract effectively ameliorated BAC-induced dry eye symptoms via enhancement of cornea resilience against BAC (10 mg/ $\mathrm{kg})$-induced damage and maintenance of conjunctival goblet cells [20]. In a steroid-induced high IOP rat model of glaucoma, elevated IOP levels decreased significantly after four weeks of oral treatment with water or ethanol extracts of $C$. cicadae. The results were almost as efficient 
as the Alphagan positive control. There were significant decreases in malondialdehyde and lactate dehydrogenase levels after 28 days of administration parallel to IOP decreases and significant increases in catalase, superoxide dismutase, and glutathione peroxidase levels [21]. Glaucoma is a neurodegenerative disease characterized by the loss of retinal ganglion cells (RGCs) [22]. Inhibition of immune activity in the retina (microglia) may decrease RGC death. This study evaluated IOP decreases in animal models and then identifies the active ingredient.

\section{Materials and Methods}

\subsection{Ethanol Extract of C. cicadae Mycelium (CCME) Preparation}

C. cicadae (BCRC MU 30106) deposited in Bioresources Collection and Research Center (BCRC) in Food Industry Research and Development Institute (Hsinchu, Taiwan) was incubated in a medium composed of $2 \%$ glucose, $1 \%$ yeast extract, and $1 \%$ soybean powder adjusted to $\mathrm{pH} 6.0$ at $25{ }^{\circ} \mathrm{C}$ on a rotary shaker $(120 \mathrm{rpm})$ for 3 days. The fermentation process was scaled up from $1.0 \mathrm{~L}$ into $200 \mathrm{~L}$ fermenter with the same medium for 5 days and then was transferred into a 20-Ton fermenter (working volume 16 Ton) for 7 days in its final production. After culturing for seven days at $25^{\circ} \mathrm{C}$, the broth was heated to $100{ }^{\circ} \mathrm{C}$ for $1 \mathrm{~h}$ and concentrated in vacuo at $55{ }^{\circ} \mathrm{C}$; it was then freeze dried, ground to a powder, and stored at $4{ }^{\circ} \mathrm{C}$ for use. The $\mathrm{C}$. cicadae mycelium powder was extracted with $95 \%$ ethanol $(1: 10, w / v)$ at room temperature for $1 \mathrm{~h}$. The extract was filtered through Whatman No.4 filter paper, and the rotor was evaporated to obtain the dried extract (CCME). CCME was suspended into various concentrations in soybean oil/water (1:1) mixture and gavaged into rabbit and rat at $1.25 \mathrm{~mL} / \mathrm{kg}$ body weight.

\subsection{High-Performance Liquid Chromatography (HPLC) Analysis}

Analysis was performed on HPLC (Hitachi 5000 series system) equipped with UV wavelength detector set at $254 \mathrm{~nm}$, and a reverse-phase column (Inert ${ }^{\circledR}$, ODS-2, $250 \mathrm{~mm} \times 4.6 \mathrm{~mm}$, $5 \mu \mathrm{m}$ ) was used with a column oven at $40^{\circ} \mathrm{C}$. The mobile phase was composed of water (A) and acetonitrile (B) with a gradient as follows: 0-15 $\min 100 \% \mathrm{~A} ; 15-40 \mathrm{~min} 100-80 \% \mathrm{~A}$; 40-55 $\min 80-5 \%$ A; 55-75 min 50-0\% A; 75-90 min 0\% A; 90-91 min 0-100\% A; 91-100 min $100 \% \mathrm{~A}$. The flow rate was kept at $1.0 \mathrm{~mL} / \mathrm{min}$, and the injection volume was $10 \mu \mathrm{L}$. Identification of adenosine and HEA was determined by comparing retention times with the standards (Sigma-Aldrich, St. Louis, MO, USA). CCME was dissolved in 20-fold volumes of $95 \%$ ethanol and filtered through a $0.45 \mu \mathrm{m}$ filter before HPLC analysis.

\subsection{Liquid Chromatography-Quadrupole-Time of Flight Mass Spectrometer (LC-QTOF/MS) Analysis}

The mass spectrometer was operated in the positive ion mode (QQQ/MS model: API 3000, Applied Biosystem, Waltham, MA, USA). The nebulizer gas, curtain gas, collision gas, ion spray voltage, and source temperature were set at 8 psi, 7 psi, 2 psi, 4500 volts, and $350{ }^{\circ} \mathrm{C}$, respectively. The mobile phase was composed of water (A) and acetonitrile (B). Separation was optimized using a gradient method with mobile phase A/B set to $95 \% / 5 \%$ from 0.00 to $5.00 \mathrm{~min}$ and $0 \% / 100 \%$ from 5.00 to $10.00 \mathrm{~min}$ and then back to $95 \% / 5 \%$ from 13.50 to $15.00 \mathrm{~min}$. the flow rate was $300 \mu \mathrm{L} / \mathrm{min}$, and the sample injection volume was $10 \mu \mathrm{L}$. The mass spectrometer was operated in the positive ion mode. The nebulizer gas, curtain gas, collision gas, ion spray voltage, and source temperature were 8 psi, 7 psi, 2 psi, 4500 volts, and $350^{\circ} \mathrm{C}$, respectively.

\subsection{Microbead Occlusion in Rat Model}

Male SD rats were obtained from the National Laboratory Animal Center (Taipei, Taiwan). The rats (170-200 g) were maintained in rat cages with standard atmospheric conditions of $12 \mathrm{~h}$ light and dark periods at $25 \pm 0.5{ }^{\circ} \mathrm{C}$ with a relative humidity of $60 \pm 5 \%$. All methods were carried out in accordance with relevant guidelines and regulations and were approved by the ethical committee of Hualien Tzu Chi Hospital (106-02). A $10 \mu \mathrm{L}$ suspension containing $30 \mathrm{mg} / \mathrm{mL}$ gamma-irradiated $8 \mu \mathrm{m}$ magnetic mi- 
crospheres in Hank's balanced salt solution (HBSS) was injected into the anterior chamber of the left eye via a 33-gauge bevel needle. The IOP was measured five times with the Icare $^{\circledR}$ TONOLAB tonometer (iCare, Vantaa, Finland) before injection and daily after use. CCME (100 mg/kg/b.w.) was gavaged once daily for 56 days. All data are expressed as mean \pm SEM. All frozen sections of the retinas were cut into 1 to $2 \mathrm{~mm}$ thickness from the ON head, to ensure the use of equivalent fields for comparison [23]. A TUNEL assay (DeadEndTM Fluorometric TUNEL System, Promega Corporation, Madison, WI, USA) was performed to detect apoptotic cells. The TUNEL-positive cells in the RGC layer of each sample were counted in 10 high-powered fields (HPF, 400× magnification).

The detailed procedure has been previously described in a previous report (Tsai et al. 2008). At least five randomly chosen areas (each $62,500 \mu \mathrm{m}^{2}$ ) in the central and midperipheral regions of each retina and their averages were estimated as the mean density of RGCs per retina. The retinas were examined with a $400 \times$ epi-fluorescence microscope (Axioskop; Carl Zeiss Meditech, Inc., Thornwood, NY, USA) equipped with a filter set (excitation filter $=350-400 \mathrm{~nm}$; barrier filter $=515 \mathrm{~nm})$, a digital camera (Axiocam MRm), and software (Axiovision 4.0). All data are expressed as mean \pm SEM.

\subsection{ROCK Kinase Assay}

The ROCK kinase assay is a luminescent kinase assay that measures IOP formed from a kinase reaction. ADP is converted into ATP and is converted into light by Kinase-Glo Luciferase. The Kinase-Glo Assay can be used to monitor the activity of virtually any ADP-generating enzyme. The relative light unit (RLU) without test compound was set as $100 \%$ (blank value), and that without enzyme and compound was set as $0 \%$ (normal value). The reaction rate (\% of black) was then calculated from the RLU.

The following reagents were used in this assay: ROCK1 (750 ug/mL, Carna Biosciences, Cat.01-109); long S6 kinase substrate peptide (Millipore, Cat\#12-420); magnesium chloride solution (Sigma, 60142-100 mL); EGTA solution (0.5 M pH 8.0, Bioword, Cat. 4052008-1); Trizma ${ }^{\circledR}$ hydrochloride solution (1 M, pH 7.5 Sigma, T2319-100 mL); bovine serum albumin solution ( $20 \mathrm{mg} / \mathrm{mL}$ in water, Sigma); Kinase-Glo luminescent kinase assay (Promega, RV6712); ripasudil (K115) hydrochloride dehydrate (Selleckchem, S7995-5 mg); ATP solution (100 mM, GE Healthcare, Cat.27-2056-010). A ROCK kinase inhibitor k-115 was used as the positive control.

Inhibition $(\%)=\frac{100-\{(\text { activity of Enzyme with Test Cmpd \& Sbustrate }- \text { Min })\} \times 100}{\operatorname{Max}-\operatorname{Min}}$

Max = observed enzyme activity measured in the presence of enzyme, substrate(s), and cofactors utilized in the method.

Min = Normal value in the method

\subsection{MLC Phosphorylation Inhibition ( $p$ MLC) Assay}

Myosin light chain2 (MLC2) is also known as myosin regular light chain (MRLC). ROCK could phosphorylate ser19 of MLC2 of smooth muscle. ROCK inhibitors could lower IOP by increasing aqueous humor outflow through the trabecular meshwork (TM). The A7r5 cell (Rat muscle cell, ATCC CRL-1444) was purchased from American Type Culture Collection (Manassas, VA, USA) and was maintained in DMEM medium supplemented with $10 \%$ fetal bovine serum and $1 \%$ penicillin-streptomycin antibiotics at $37{ }^{\circ} \mathrm{C}$ in a humidified atmosphere of $5 \% \mathrm{CO}_{2}$. Cells were seeded at $6^{*} 10^{5}$ cells per well in a P100 dish and incubated overnight.

Cells were changed to a new medium prior to treatment. Cell lysis and Western blots were performed by following the usual protocol. CCME prepared in 100\% DMSO as a stock solution was added to cell lysate at various concentrations. AR-13324 was used as the positive control. The cell lysate was assayed with pMLC2 (Thr19/Ser19) antibody (Cell Signaling, CST3674) in HaltTM protease and phosphatase inhibitor (Thermo Fisher 78442, Waltham, MA, USA). GAPDH (Sigma), HRP goat anti-rabbit (Jackson Immuno Research, 
111-035-003, West Grove, PA, USA), skim milk, and SuperSignal West Pico Maximum Sensitivity substrate (Thermo Fisher, 34080) were used for the Western blot.

\subsection{Normal Rabbit Model}

For this study, 8-12-week-old female New Zealand White (NZW) rabbits were purchased from HUEI-JYUN Company (Changhua, Taiwan). The animals were housed in the rabbit laboratory of Master Laboratory Co., Ltd. During the study, data of the animals including cage number, strain, weeks old, animal I.D., date of animal arrival, experimental number, group, and period were noted on the housing card. The housing conditions were kept in $12 \mathrm{~h}$ light and $12 \mathrm{~h}$ dark at $23 \pm 2{ }^{\circ} \mathrm{C}$ and $40-70 \%$ humidity. The animals were given free access to food and water. To ensure the health of the animals, clinical observations were noted daily by veterinarians from Master Laboratory Co., Ltd. and Industrial Technology Research Institute (ITRI), Hsinchu, Taiwan, during quarantine and experiments, respectively. The animals were placed in this study after one week of quarantine. The experimental protocol listed below has been reviewed and approved by the Institutional Animal Care and Use Committee (IACUC-2016-040) of ITRI. In total, 25 rabbits were randomly divided into five groups (blank, $0.5 \%$ Timolol via eye drop; vehicle, $2.5 \mathrm{mg} / \mathrm{kg} / \mathrm{b} . \mathrm{w}$. and $25 \mathrm{mg} / \mathrm{kg} / \mathrm{b}$.w. CCME oral); one drop of $0.5 \%$ Timolol (Timoptol ${ }^{\circledR}$ Ophthalmic Solution $0.5 \%: 0.5 \%$ timolol maleate, MSD) was applied to the right eye of each rabbit per day as a positive control.

The IOP of the right eye was measured five times with Tono Vet Tonometer type Tv01.

$$
\Delta \mathrm{IOP}=\mathrm{IOP} \text { time point }-\mathrm{IOP} 0 \mathrm{~h}
$$

$$
\text { Percentage of } \Delta \mathrm{IOP}=\Delta \mathrm{IOP} / \mathrm{IOP} 0 \mathrm{~h}
$$

Data are presented as the mean \pm S.E.M. (standard error of mean). Differences between groups were analyzed with Student's two-tailed $t$-test; $p<0.05$ was considered significant.

\subsection{Dextrose-Induced Acute Glaucoma Model}

Three male (2.8-3.0 kg) and three female (3.0-3.6 kg) New Zealand white rabbits were purchased from Weishinhun company (New Taipei City, Taiwan) and were quarantined and housed under standard conditions ( $12 \mathrm{~h}$ dark, $12 \mathrm{~h} \mathrm{light,} 20^{\circ} \mathrm{C}, 55 \pm 15 \%$ humidity) for seven days before the experiment. All producers were conducted under the SOP (SOPA-303, SOPA-203, SOPA-315, and SOPA-206) of SuperLab (New Taipei City, Taiwan). Rabbits were given free access to Altromin 2023 diet and R.O. water. Every rabbit had its own cage. They were randomly divided into three groups-one male and one female per group. The same dose of soybean oil/water mixture was given as the control group. IOP was measured with Tono-Pen VETTM Tonmeter type without anesthesia. Here, $5 \%$ glucose solution $(15 \mathrm{~mL} / \mathrm{kg}$ body weight) was injected through the marginal ear vein to rapidly induce ocular hypertension. A repeat experiment was conducted with the same rabbits after a one-week washout. Values are expressed as the means \pm standard deviation (SD). The Mann-Whitney $U$ test was used to compare the mean value between the control group and the CCME-treated group. Statistical significance was defined as a $p$-value $<0.05$.

\section{Results}

\subsection{Glaucoma Model}

Normal rat IOP values are $10-12 \mathrm{mmHg}$. A $10 \mathrm{uL}$ magnetic microbeads suspension was injected into anterior chambers of the right eye to block the outflow of the aqueous humor (AH) at day 0 (Figure 1). The IOPs of both control and CCME groups before surgery were recorded as $10.8 \pm 0.91$ and $10.8 \pm 1.22 \mathrm{mmHg}$, respectively. After surgery, $1.25 \mathrm{~mL} / \mathrm{kg}$ b.w. CCME and vehicle were gavaged on the same day and for the subsequent 56 days once daily. On day 1, a substantial increase in IOP from day 0 in both control $(25.4 \pm 3.2 \mathrm{mmHg})$ and CCME groups $(23 \pm 1.52 \mathrm{mmHg})$ were observed. The CCME group IOP was lower than the control group, but the difference was not significant. Continuously 
higher IOPs in the control were seen from day 1 to day $56(25.4 \pm 3.2 \mathrm{mmHg})$ versus the CCME. Compared with the sustained high IOPs of control, the CCME had a lower IOP (23 mmHg) at day $2(22.1 \pm 1.91 \mathrm{mmHg})$. This further decreased the IOP on day 3 $(18.2 \pm 2.04 \mathrm{mmHg})$ versus the control group $(p<0.05)$. The IOP decreased and remained at $17.3 \pm 1.70 \mathrm{mmHg}$ for the next 53 days. These data suggest a significant alleviation of CCME when IOP increases. The $\triangle \mathrm{IOP}$ between control and CCME groups progressively increased, but the alleviated IOP in CCME still did not reach baseline values $(10.8 \mathrm{mmHg})$ even after 56 days. On day 56, the IOP of the CCME group was $60.5 \%$ lower than the control group (26.5-17.3 $\mathrm{mmHg}) /(26.5-10.8 \mathrm{mmHg}) \mathrm{mmHg})$.

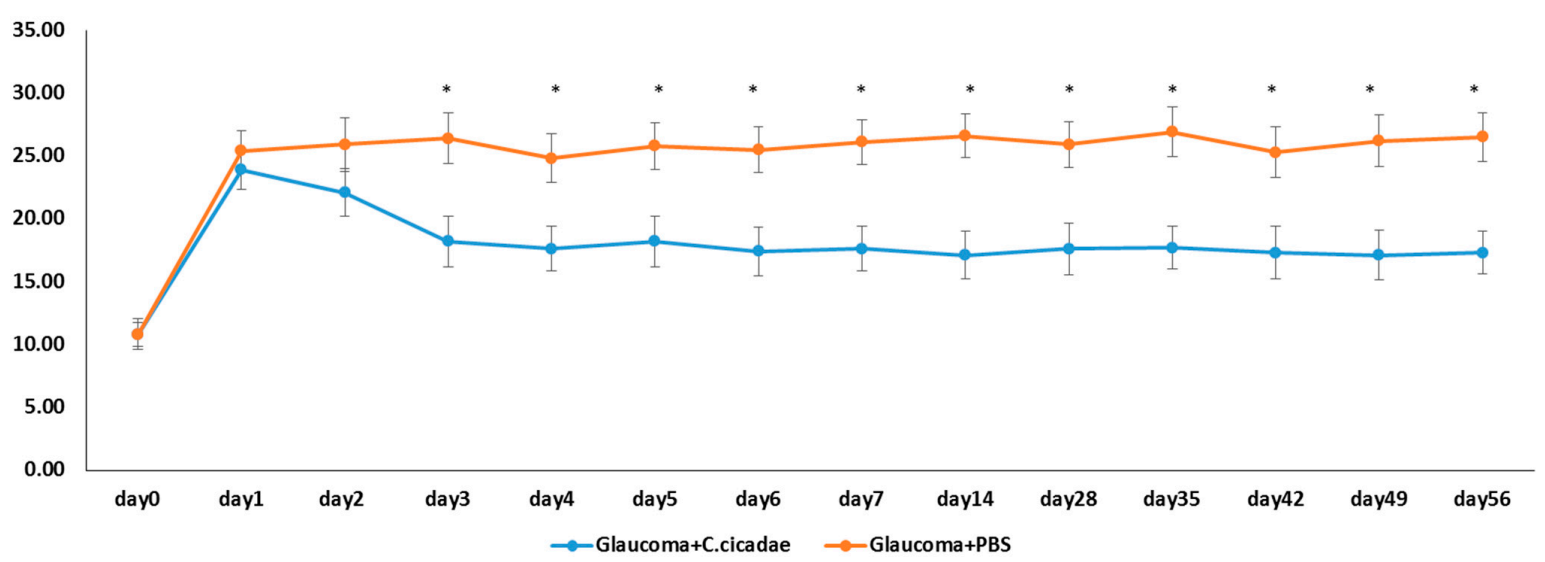

Figure 1. Effect of C. cicadae mycelia extract on IOP in the glaucoma model. ${ }^{*}: p<0.05, n=12$, mean \pm SEM. versus Glaucoma+PBS group.

There was mass mortality due to the impairment of microbeads. Terminal deoxynucleotidyl transferase (TdT) dUTP nick-end labeling assay was performed using a microscope (Figure 2a). The corresponding IOP reduction led to apparent protection of CCME in RGCs mortality versus control. Nearly $77.2 \%$ of RGCs were protected from death (Figure $2 b$ ).
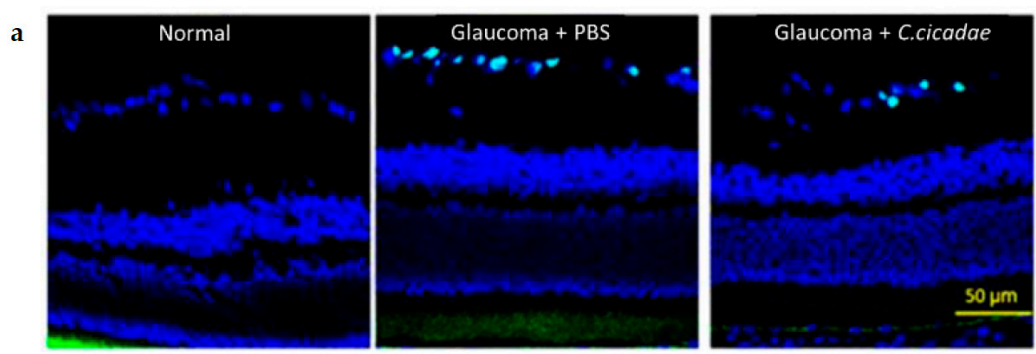

b

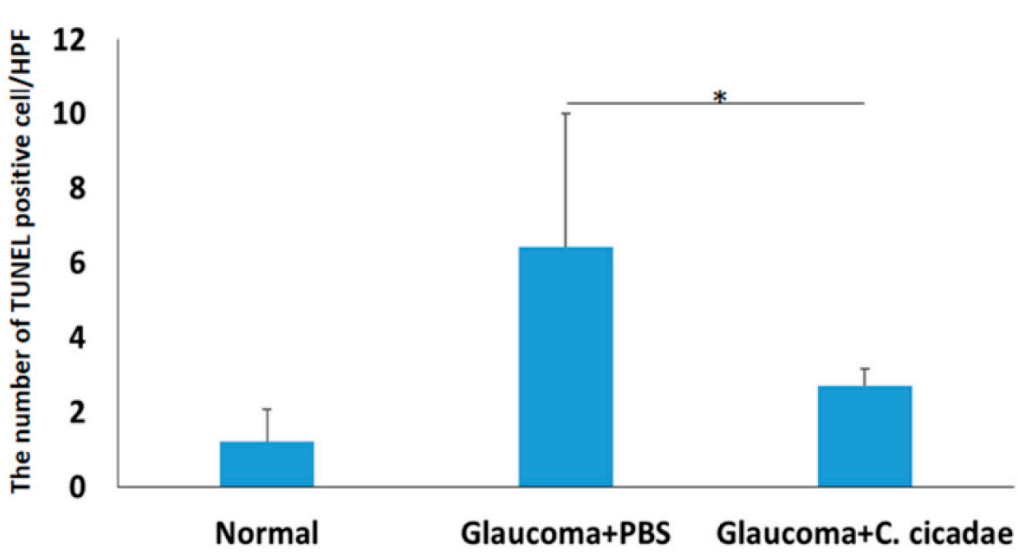

Figure 2. The apoptosis of retinal ganglion cells (RGCs) of rats at day 56. (a) Representative terminal deoxynucleotidyl transferase dUTP nick-end labeling (TUNEL) staining of day 56. (b) The number of TUNEL-positive cells in the RGC. (mean \pm standard error of the mean, SEM; ${ }^{*} p<0.05$ ). 


\subsection{ROCK Kinase Assay}

The IOP reduction may be caused by a decrease in AH outflow resistance or a decline in $\mathrm{AH}$ formation. Rho-associated kinase (ROCK) is a critical serine/threonine kinase Rho GTPase. ROCK inhibitions were reported to have a lower IOP value versus increasing the aqueous outflow through the TM. Rock kinase inhibition and myosin light chain (MLC) phosphorylation confirmed the inhibitor activity in CCME.

Both doses of CCME $(0.25$ and $2.5 \mu \mathrm{g} / \mathrm{mL})$ showed dose-dependent but not significant inhibition ( $4 \pm 4$ and $29 \pm 4 \%$ ) versus K-115 (M.W. 323.4; Table 1). This positive control reduced ROCK kinase activity at IC50:21.4 $\pm 1.6 \mathrm{nM}$. Figure 3 compares the abilities of controls and CCME to reduce MLC phosphorylation level in the cell line model. A high dose $(25 \mu \mathrm{g} / \mathrm{mL})$ of CCME could reduce $59.0 \%$ phosphorylation and was dose-dependent but had poor potential versus IC50: $1 \mathrm{nM}$ for AR-13324 (M.W.526.4).

Table 1. ROCK kinase inhibition assay for CCME.

\begin{tabular}{cc}
\hline Conc & Inhibition of \% \\
\hline CCME $0.25 \mu \mathrm{g} / \mathrm{mL}$ & $4 \pm 4$ \\
CCME $2.5 \mu \mathrm{g} / \mathrm{mL}$ & $29 \pm 4$ \\
CCME $25 \mu \mathrm{g} / \mathrm{mL}$ & $4 \pm 2$ \\
K-115 $21.4 \pm 1.6 \mathrm{nM}$ & 50 \\
\hline
\end{tabular}
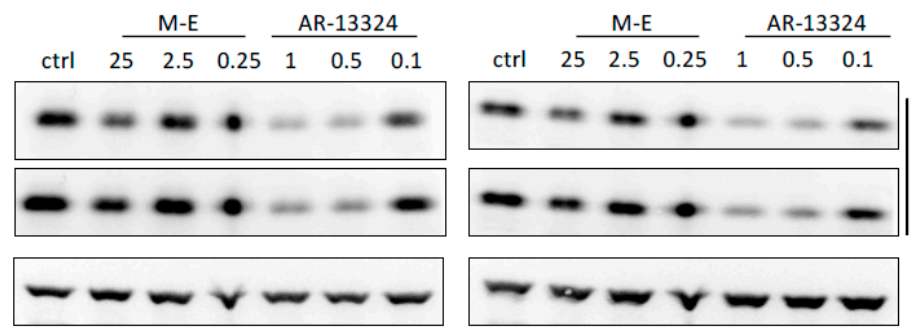

IB: pMLC2

(different exposure time)
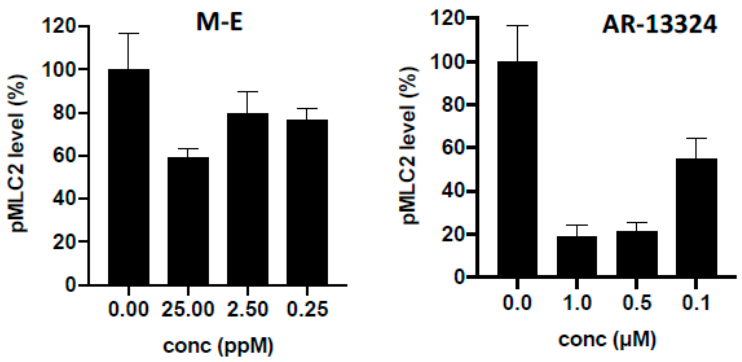

Figure 3. pMLC2 inhibition for CCME and ROCK inhibitor AR-13324 by Western blot.

\subsection{Normal Rabbit Model}

NZW rabbits with a normal IOP were tested to realize an IOP-reduced effect of CCME on cross-species animals. Timolol eye drops served as a positive control. CCME was fed orally, and IOP was measured at $0,1,3$, and $24 \mathrm{~h}$ on day 1 and day 2 to match Timolol's optimal function time. Both Timolol and high dose CCME immediately lowered the IOP at $1 \mathrm{~h}(-2.5$ and $-1.5 \mathrm{mmHg})$, with a slight increase at $3 \mathrm{~h}$. This then returned to baseline IOP at $24 \mathrm{~h}$ (Figure $4 \mathrm{a}, \mathrm{b})$.

The repeatability of IOP decrease with an increase in back-to-normal conditions on day 2 was observed in both Timolol and CCME groups. CCME also had a dose dependence on day 1 and day 2. Milder IOP decreases (12\%) were seen for high-dose CCME. This is less effective than $20 \%$ for Timolol at $1 \mathrm{~h}$, but the convenience of the oral route and the quick responses still potentialized these applications. 

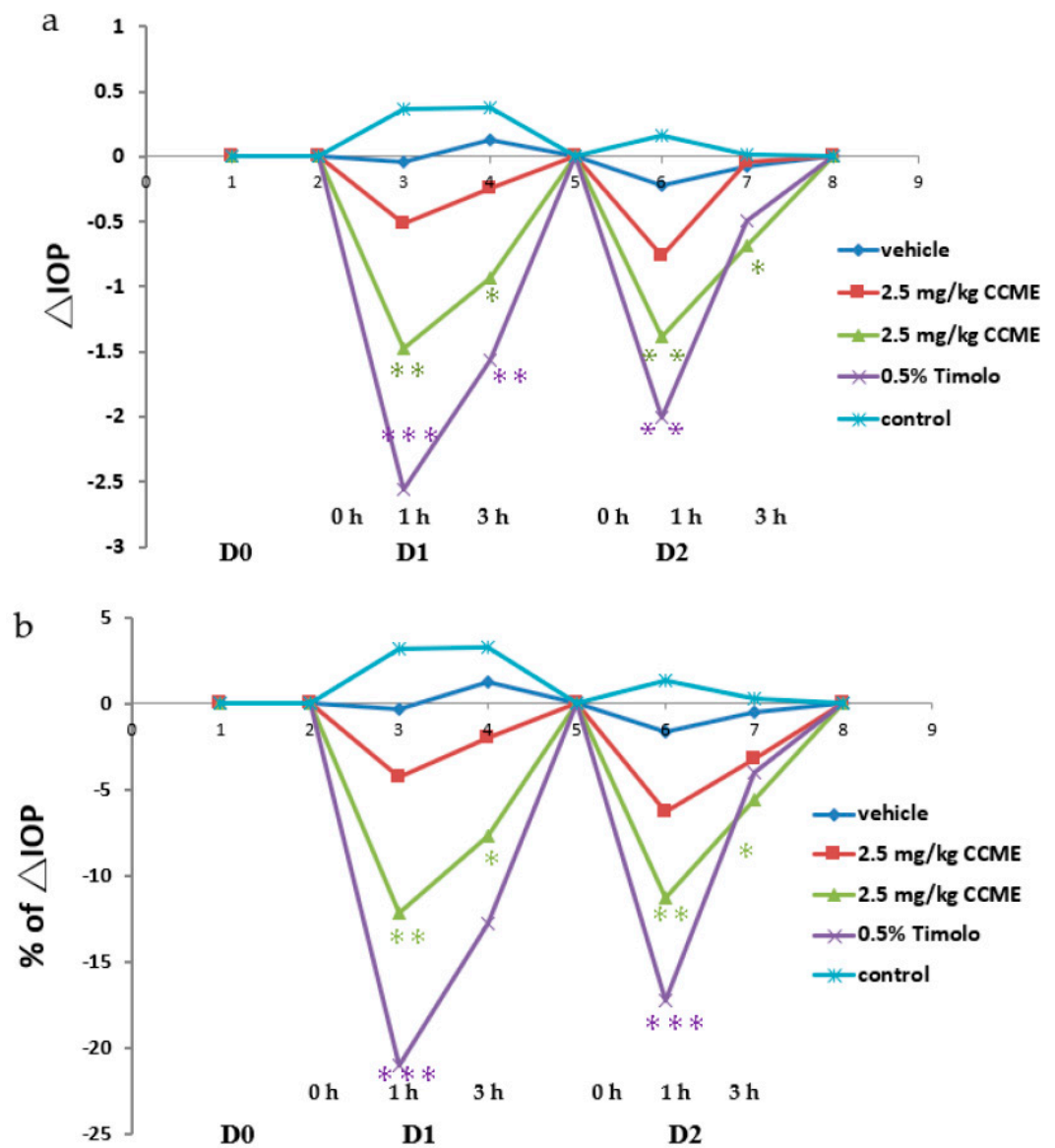

Figure 4. Effect of $C$. cicadae mycelia extracts on IOP in a rabbit model: (a) the $\triangle \mathrm{IOP}$ (IOPtime-IOP0) of time course on the normotensive rabbit eye model. Dark blue is vehicle, light blue is control, red is $2.5 \mathrm{mg} / \mathrm{kg}$ CCME, green is $25 \mathrm{mg} / \mathrm{kg} \mathrm{CCME}$, and purple is Timolol. ${ }^{*} p<0.05,{ }^{* *} p<0.01$ and *** $p<0.001$ versus vehicle control group; (b) the percentage of $\triangle \mathrm{IOP}$ on the ocular normotensive rabbit model. Dark blue is vehicle, light blue is control, red is $2.5 \mathrm{mg} / \mathrm{kg}$ CCME, green is $25 \mathrm{mg} / \mathrm{kg}$ CCME, and purple is Timolol.

\subsection{Dextrose-Induced Acute Glaucoma Model}

We pathologically studied acute high IOP rabbit models induced by posterior intravenous administration of 5\% dextrose infusion. The IOP sharply increased, from 23.7 to $38.4 \mathrm{mmHg}(n=4)$, at $15 \mathrm{~min}$ after dextrose infusion and then gradually decreased to initial IOP at 90 min (Figure 5). CCME requires one hour for activity (Figure 4); the pretreatment of CCME at $-60 \mathrm{~min}$ was designed to function between 0 and $90 \mathrm{~min}$. Compared with the vehicle, CCME obviously displayed less of a rise in IOP from 15 to $90 \mathrm{~min}$. Here, a 25.96\% reduction $(37.75 \rightarrow 29.95)$ in IOP was seen with a 53.35\% reduction (29.95-23.4)/(37.75-23.4) in IOP elevation at $15 \mathrm{~min}$ for high-dose CCME. There was less area under the curve (AUC) versus control. For CCME, the IOP at time 0 min before infusion was nearly the same as that at time $-60 \mathrm{~min}$, indicating that the time required for CCME is at least $60 \mathrm{~min}$.

\subsection{CCME Active Components}

The fingerprint chromatogram contains two specific active components purified from the liquid fermentation of CCME (retention time $27.7 \mathrm{~min}$ and $30.8 \mathrm{~min}$ ), as shown in Figure 6a. These compounds' structures were confirmed using the liquid chromatography in combination with tandem MS (LC-QTOF/MS) operating in multiple reaction monitoring $(\mathrm{MRM})$ mode by identifying precursor $\rightarrow$ production transitions (Figure $6 \mathrm{~b}$ ). 

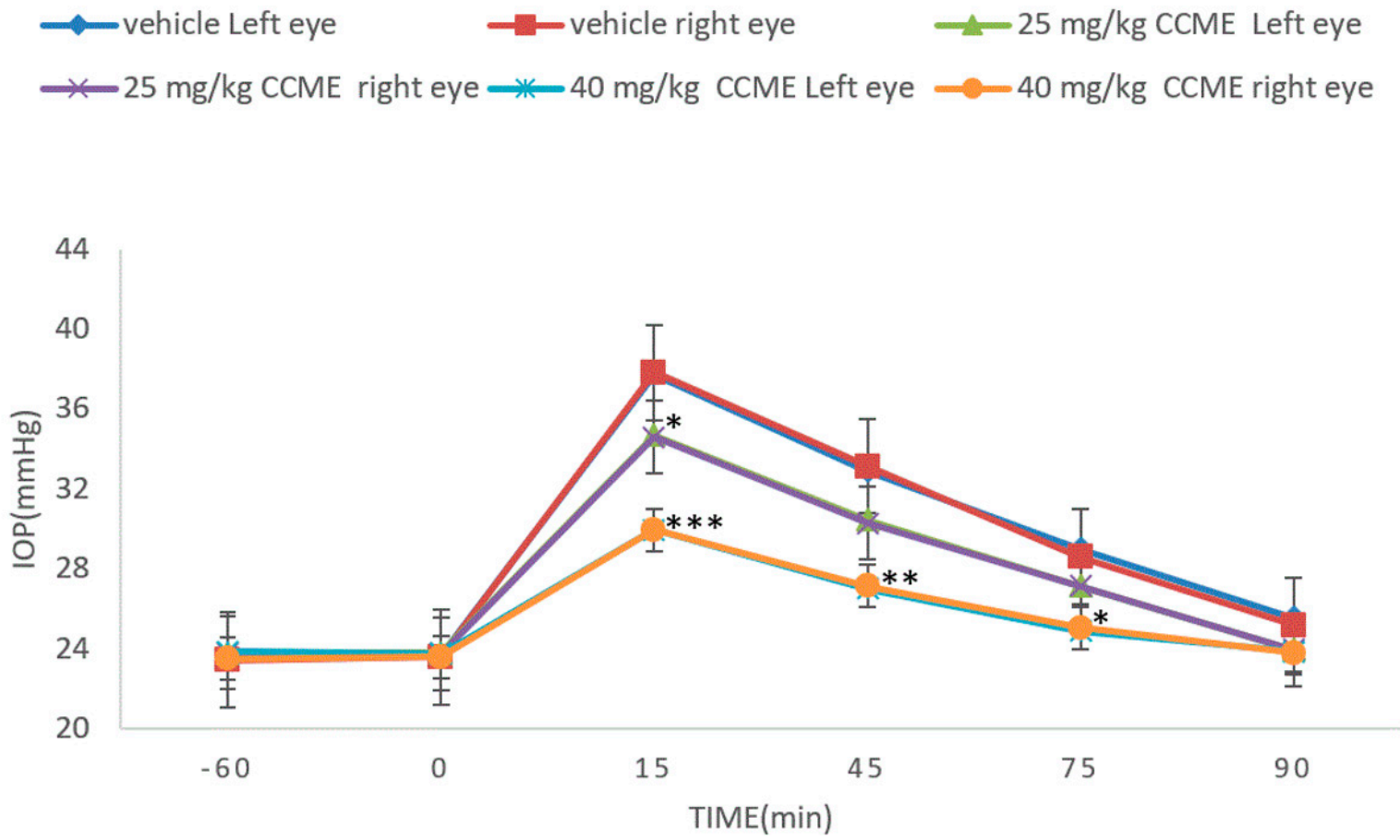

Figure 5. Anti-hypertensive effect of $C$. cicadae mycelia extract (CCME) in dextrose-induced acute glaucoma rabbit (A45-25 mg/kg/b.w.; B—40 mg/kg/b.w.) $n=4$, mean \pm SEM. ${ }^{*} p<0.05,{ }^{* *} p<0.01$ and ${ }^{* * *} p<0.001$, compared with solvent control group.

a

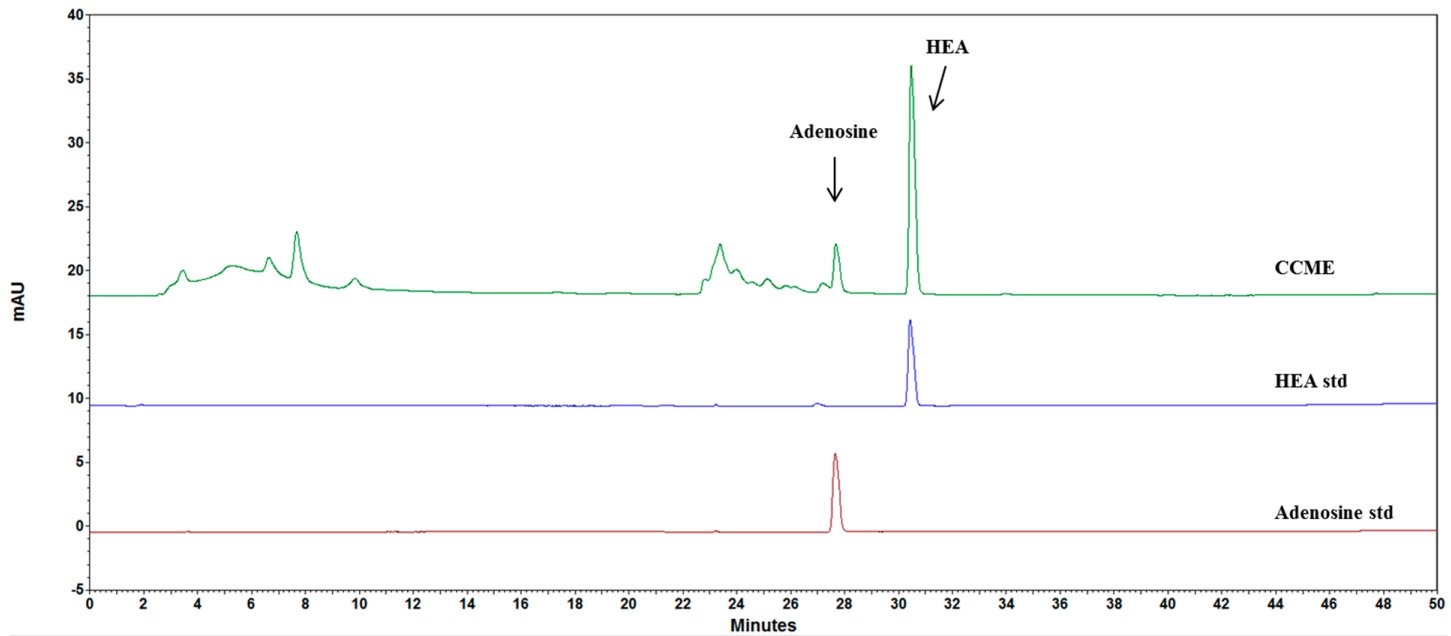

b adenosine

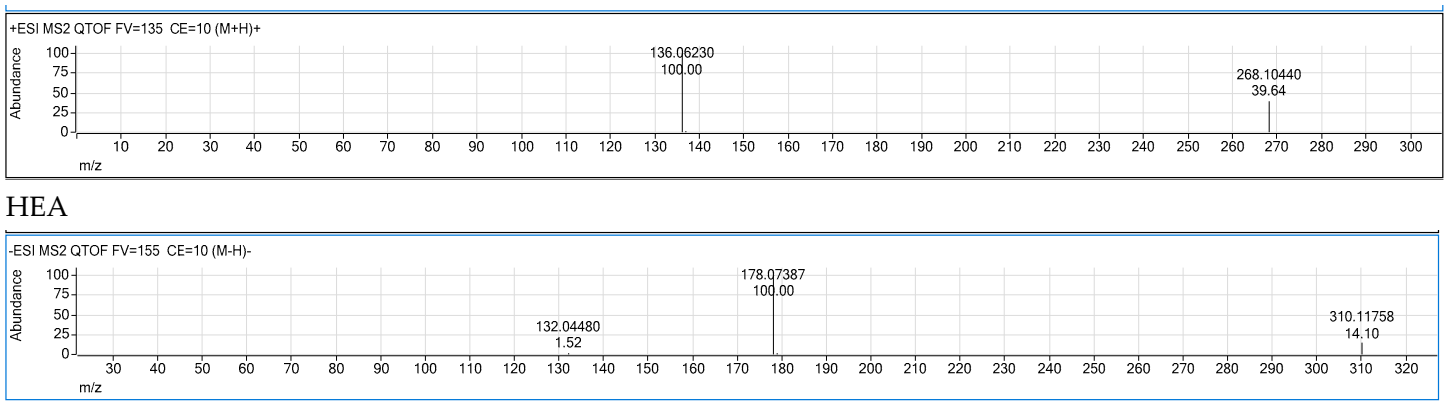

Figure 6. (a) HPLC of adenosine and HEA extracted from $C$. cicadae with retention times labeled at $27.7 \mathrm{~min}$ for adenosine and $30.8 \mathrm{~min}$ for HEA. Twenty microliters of adenosine or HEA; (b) LCQTOF/MS spectra of adenosine with parental ion detected at $m / z$ 268.10440; HEA with parental ion detected at $m / z 310.11758$. 
Concentrations of adenosine and HEA were determined as 2.2 and $5.1 \mathrm{mg} / \mathrm{g}$, respectively. Adenosine has been shown to have low bioavailability due to fast metabolism in vivo [24]. HEA is an adenosine derivative and has anti-hydrolytic capability from adenosine deaminase (ADA) hydrolysis (data not shown). HEA was chosen as a potential candidate to assess its IOP reducing activity. HEA significantly mitigated the IOP increase similar to CCME (Figure 7). Relative to the vehicle, there was a nearly $26.04 \%$ reduction $(38.4 \rightarrow 28.4)$ in IOP and $68.5 \%$ reduction $(28.4-23.8) /(38.4-23.8)$ in IOP elevation at $15 \mathrm{~min}$ in both eyes in the high dose group $(0.2 \mathrm{mg} / \mathrm{kg}$ body weight). Dose-dependent effects were also noted at all sampling times.
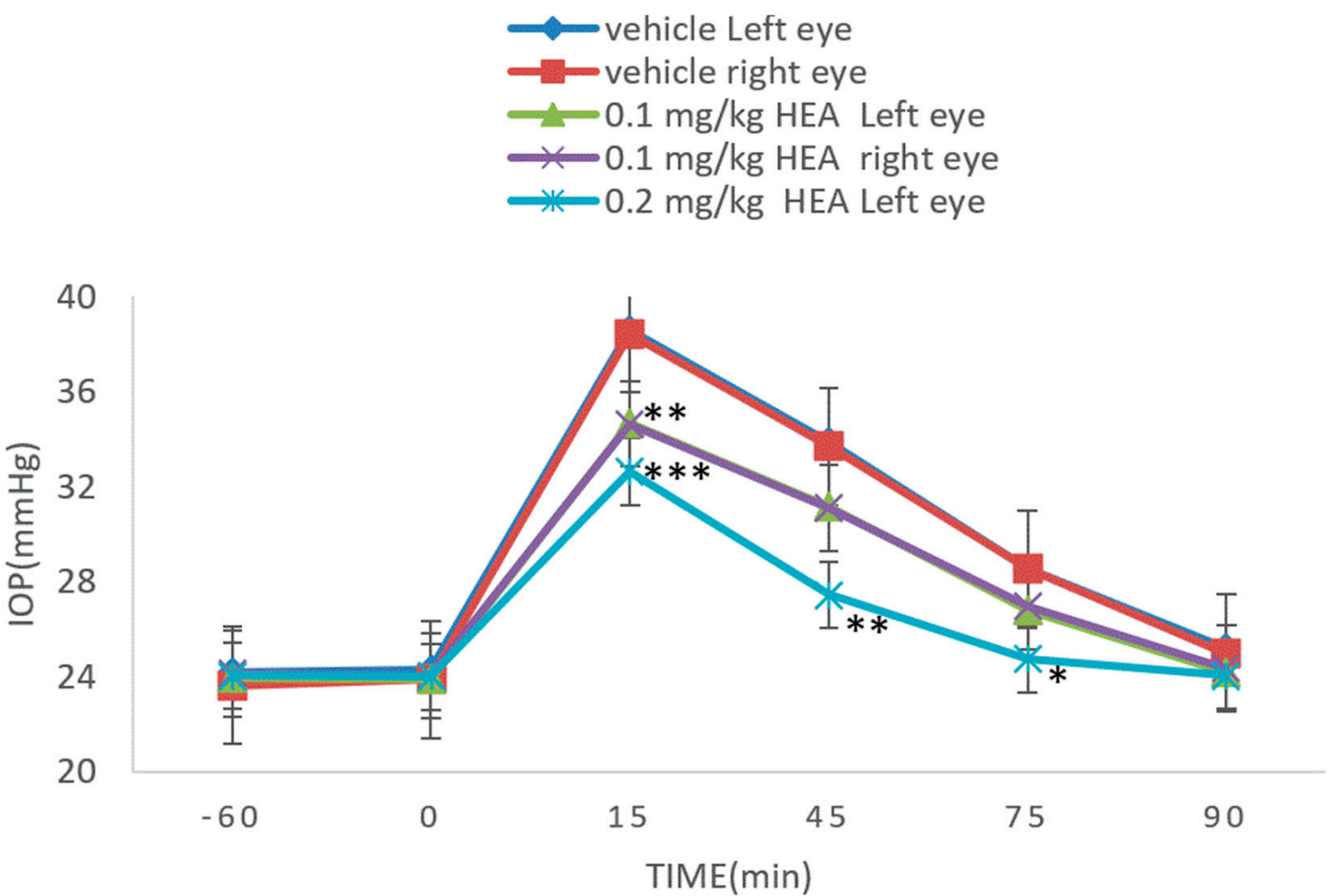

Figure 7. Anti-hypertensive IOP effect of HEA in dextrose induced acute glaucoma rabbit $(\mathrm{A}=0.1 \mathrm{mg} / \mathrm{kg} / \mathrm{b} . w ., \mathrm{B}=0.2 \mathrm{mg} / \mathrm{kg} /$ b.w., $n=4) .{ }^{*} p<0.05,{ }^{* *} p<0.01$ and ${ }^{* * *} p<0.001$, compared with solvent control group.

\section{Discussion}

According to the $2020 \mathrm{WHO}$ study, almost one billion people are afflicted with vision impairment including glaucoma, retinal inflammation, dry eye, diabetic retinopathy, and age-related macular degeneration $[25,26]$. Glaucoma is the second most frequent cause of irreversible blindness in the world and the global burden is predicted to be 112 million in 2040 [27]. Glaucoma is a progressive loss of RGCs, optical nerve head, and retinal nerve filter layers resulting in visual field deficiencies. Glaucoma caused by optical nerve damage is related to IOP increase. Axon degeneration of RGCs at the optic nerve head results from high IOP and is parallel to the apoptosis of RGCs. This is believed to be a leading risk factor for the onset and progress. Lowering IOP can protect the nerve and reduce the development and progression of glaucoma.

C. cicadae mycelium extracts (water and ethanol) can significantly reduce the steroidinduced high IOP in rats similar to Alphagram. This study confirmed the IOP-dropping effect in various models and animal species. The microbead occlusion model was used to induce ocular hypertension in rats. A microbead suspension was injected into the anterior chamber to occlude aqueous humor outflow and to raise the IOP $135.1 \%$ (from 10.8 to $25.4 \mathrm{mmHg}$ ) at day 1 for the control group. Compared with control, CCME decreased 
the IOP $(25.4 \pm 3.9 \mathrm{mmHg})$ at day 1 ; the IOP gap between both groups continued to increase until day $3(26.4 \pm 18.2 \mathrm{mmHg})$ and stabilized until day $56(26.5 \pm 1.95 \mathrm{mmHg}$ vs. $17.3 \pm 1.7 \mathrm{mmHg})$.

During these 56 days, the IOPs in CCME-treated animals were always higher than those of initial IOP $(10.8 \pm 1.22 \mathrm{mmHg})$ on day 0 before bead injection. Microbeads are difficult to degrade, and these data implied that CCME's partial dropping function may arise from creating more leakages between the bead and trabecular meshwork (TM) cells or reducing aqueous humor $(\mathrm{AH})$ production by the ciliary body epithelium. Glaucoma is a chronic neuropathy mostly associated with elevated IOP. In this experiment, there were 56 days between model induction and RGC impairment under high IOP. After sacrifice at day 56, dead RGCs were evaluated with a TUNEL assay. A higher IOP indeed led to much more apoptosis in RGCs in the vehicle and CCMC groups than in the sham group (Figure 2). According to the report of Nakazawa et al. (2006), elevated IOP would stimulate the Fas ligand binding to the death receptors and result in apoptosis in RGCs [27]. The main cause of vision loss in glaucoma is apoptosis in RGCs. Significant reductions in CCME in apoptosis are attributed to lower IOP values. CCME protected 77.2\% RGCs from mortality by mediating a lower IOP versus control. Bradely et al. (1998) discovered the contractile properties of TM cells. Increasing the contraction of TM cells in glaucoma may result in elevated AH outflow resistance [28]. Disruption of TM actin cytoskeleton resulting in reducing outflow resistance has also been observed by Tian et al. [29,30]

Rhopressa $^{\circledR}$ (netarsudil ophthalmic solution, Aerie Pharmaceuticals) is a rho kinase (ROCK) inhibitor and was approved by the FDA in late 2017 for reducing IOP in primary open-angle glaucoma or ocular hypertension patients by increasing the AH outflow. Its molecular mechanism was believed to decrease actin-myosin contraction, with reduced actin stress fibers and focal adhesion in TM [26].

CCME was evaluated with ROCK assay to confirm its possible inhibitory mechanism. Table 1 and Figure 3 show that no significant inhibition was found for CCME even up to $25 \mathrm{mg} / \mathrm{mL}$. This inhibiting concentration is far more than those of positive controls. Moreover, CCME was given orally. The concentration would be further diluted before it reached TM cells in vivo. These data implied that the IOP decreasing effects are not caused by ROCK inhibition.

Adenosine receptors (ARs) are well repressed in all the ocular tissues such as TM and retina. ARs are implicated in retinal function, neuronal survival, and blood flow. Many studies have focused on the role of ARs on AH formation and outflow facility, IOP, and optic nerve protection. AR agonists and antagonists have also been reported to modulate aqueous humor formation, outflow facility, IOP modulation, and optic neuroprotection [31]. Some of these are exploited in clinical trials for glaucoma and dry eye disease [32]. Many AR agonists and antagonists have been evaluated as potential therapeutic agents in glaucoma. However, there are different effects of the AR agonists and antagonists in different animal species. The cross-species difference should be evaluated.

Aside from rats, normal NZW rabbits were fed with CCME to check their IOP mitigation. Timolol is a $\beta$-adrenergic antagonist (beta blocker). These eye drops can treat glaucoma, and therefore, they served as a positive control. Timolol achieved its maximal IOP-lowering effect at 1-2 $\mathrm{h}$ after administration, and thus, sampling time was set at 0 , 1,3 , and $24 \mathrm{~h}$. CCME had its optimal IOP reduction at $1 \mathrm{~h}$ with dose dependence and reproducibility for 2 days (Figure 4). It was quickly available orally. It was not as effective as Timolol, but the oral delivery has advantages in terms of compliance and side effects from preservatives in eye drops. In addition, the active ingredient purified from CCME might be used in targeted delivery.

In addition to normal rabbits, the pathologic acute high IOP rabbits induced by dextrose infusion were also studied (Figure 5). In all three groups, dextrose promptly raised the IOPs within $15 \mathrm{~min}$ with a gradual decline to baseline in $90 \mathrm{~min}$. CCME also showed significant prevention and dose dependence. At $1 \mathrm{~h}$ after administration, it is necessary for CCME to exhibit an IOP decrease in normal rabbits (Figure 5); pre-treating with CCME at 
$-60 \mathrm{~min}$ was carried out before dextrose induction. The IOPs at $-60 \mathrm{~min}$ were almost the same as those at 0 min before dextrose injection for all placebo and CCME groups. This suggests that $60 \mathrm{~min}$ is required for this activity.

The CCME ethanol extract was rich in adenosine and HEA (adenosine derivative) (Figure 6). We have never found cordycepin in C. cicadae mycelium or fruiting bodies. The roles of adenosine in IOP modulation have been well established including binding to A1AR of TM cells to reduce outflow resistance in lowering IOP and stimulating A3AR to increase the fluid inflow of AH and IOP $[33,34]$. HEA was studied because it is a known $\mathrm{Ca}^{2+}$ antagonist in inhibiting muscle contraction. The HEA structure may be legitimately considered as an AR agonist or antagonist. The assessment was conducted with the same induced model as CCME. As a result, HEA showed a significant IOP reduction with dose dependence (Figure 7). Santiago et al. (2002) mentioned that ARs exist in the ciliary body, trabecular meshwork, scleroderma, and retina. They are related to AH formation, outflow, and IOP homeostasis [35]. Zhong et al. found that neuronal survival and blood flow were also affected by ARs.

Many A1AR agonists, A2aAR agonists, and A3AR agonists are potentially therapeutic candidates for treating inflammatory diseases such as glaucoma by decreasing IOP and inducing specific anti-inflammatory effects $[36,37]$. We speculated that HEA may mediate significant effects on IOP similar to AR agonists or antagonists [38-41]. As an adenosine derivative, HEA may function as an AR agonist or antagonist in decreasing IOP and preventing RGC death in rats and rabbits. This hypothesis was partially supported by HEA activation of A1AR and A2aAR as agonists [42,43]. Activated P2X7 receptor on RGCs increases intracellular $\mathrm{Ca}^{2+}$ levels and induces cell apoptosis. Adenosine and ATP are agonists of the $\mathrm{P} 2 \mathrm{X} 7$ receptor and can activate $\mathrm{P} 2 \mathrm{X} 7$ receptors [44]. We do not know the effect of HEA on the P2X7 receptor, but it is known as a $\mathrm{Ca}^{2+}$ channel antagonist and can block the influx of $\mathrm{Ca}^{2+}$ into the cytosol. Recently, HEA was found in rat brain after $30 \mathrm{~min}$ of i.v. administration. This proved that HEA could pass through the blood-brain barrier (BBB). The CCME promptly initiated IOP reduction at $1 \mathrm{~h}$ following the oral route [19]. Studies on pharmacokinetics and blood-retina-barrier (BRB) are also needed.

\section{Conclusions}

Cordyceps cicadae mycelium alcohol extract (CCME) was shown to have IOP-reducing potential in rat and rabbit models. This study was confirmed with only minor inhibition in the phosphorylated myosin light chain 2 (pMLC2) pathway. Its active ingredient was HEA, which could significantly mitigate the IOP. CCME also protected RGCs from death, similar to the high IOP in rats. Future efforts will focus on elucidating the mechanism and possibly achieving the regulate intraocular pressure.

Author Contributions: Data analysis, writing-original draft preparation, L.-Y.L.; fermentation of C. cicadae, J.-H.H.; resources (mycelium preparation), H.-I.F.; project administration, supervision, writing-review and editing, C.-C.C.; design and coordination, K.-C.T. All authors have read and agreed to the published version of the manuscript.

Funding: This research received no external funding.

Institutional Review Board Statement: The study was conducted according to the guidelines of the Institutional Animal Care and Use Committee in Hualien Tzu Chi Hospital (106-02).

Informed Consent Statement: Not applicable.

Data Availability Statement: All data can be assessed from L.Y. Lee via the email address.

Conflicts of Interest: The authors declare no conflict of interest.

Sample Availability: Not applicable. 


\section{References}

1. Yu, H.M.; Wang, B.S.; Huang, S.C.; Duh, P.D. Comparison of protective effects between cultured Cordyceps militaris and natural Cordyceps sinensis against oxidative damage. J. Agric. Food Chem. 2006, 54, 3132-3138. [CrossRef]

2. Zhu, Y.; Yu, X.; Ge, Q.; Li, J.; Wang, D.; Wei, Y.; Ouyang, Z. Antioxidant and anti-aging activities of polysaccharides from Cordyceps cicadae. Int. J. Biol. Macromol. 2020, 15, 394-400. [CrossRef]

3. Won, S.Y.; Park, E.H. Anti-inflammatory and related pharmacological activities of cultured mycelia and fruiting bodies of Cordyceps militaris. J. Ethnopharmacol. 2005, 96, 555-561. [CrossRef] [PubMed]

4. Weng, S.C.; Chou, C.J.; Lin, L.C.; Tsai, W.J.; Kuo, Y.C. Immunomodulatory functions of extracts from the Chinese medicinal fungus Cordyceps cicadae. J. Ethnopharmacol. 2002, 83, 79-85. [CrossRef]

5. Zhang, Q.; Olatunji, O.J.; Chen, H.; Tola, A.; Oluwaniyi, O.-O. Evaluation of the Anti-Diabetic Activity of Polysaccharide from Cordyceps cicadae in Experimental Diabetic Rats. Chem. Biodivers. 2018, 15, e1800219. [CrossRef]

6. Li, L.; Zhang, T.; Li, C.; Xie, L.; Li, N.; Hou, T.; Wang, Y.; Wang, B. Potential therapeutic effects of Cordyceps cicadae and Paecilomyces cicadae on adenine-induced chronic renal failure in rats and their phytochemical analysis. Drug Des. Dev. Ther. 2018, 13, 103-117. [CrossRef]

7. Huang, Y.-S.; Wang, X.; Feng, Z.; Cui, H.; Zhu, Z.; Xia, C.; Han, X.; Liu, W.J.; Liu, Y.N. Cordyceps cicadae Prevents Renal Tubular Epithelial Cell Apoptosis by Regulating the SIRT1/p53 Pathway in Hypertensive Renal Injury. Evid. Based Complement. Alternat. Med. 2020, 2020, 7202519. [CrossRef] [PubMed]

8. Zheng, R.; Zhu, R.; Li, X.L.; Li, X.Y.; Shen, L.I.; Chen, Y.; Zhong, Y.F.; Deng, Y.Y. N6-(2-Hydroxyethyl) Adenosine From Cordyceps cicadae Ameliorates Renal Interstitial Fibrosis and Prevents Inflammation via TGF- $\beta 1 /$ Smad and NF-kB Signaling Pathway. Front. Physiol. 2018, 9, 1229. [CrossRef]

9. Wang, X.H.; Qin, A.O.; Xiao, F.; Olatunji, O.J.; Zhang, S.Y.; Pan, D.; Han, W.Z.; Wang, D.Q.; Ni, Y.H. N6-(2-hydroxyethyl)adenosine from Cordyceps cicadae protects against diabetic kidney disease via alleviation of oxidative stress and inflammation. $J$. Food Biochem. 2019, 43, e12727. [CrossRef] [PubMed]

10. Chen, Y.L.; Yeh, S.H.; Lin, T.W.; Chen, C.C.; Chen, C.S.; Kuo, C.F. A 90-Day Subchronic Toxicity Study of Submerged Mycelial Culture of Cordyceps cicadae (Ascomycetes) in Rats. Int. J. Med. Mushrooms 2015, 17, 771-781. [CrossRef]

11. Hsu, J.H.; Yeh, S.H.; Huang, W.C.; Chen, C.C. Isolation, Identification and Genotoxicity Test of Cordyceps cicadae mycelium. J. Test. Qual. Assur. 2015, 4, 114-127.

12. Jhou, B.Y.; Hsu, J.H.; Yeh, S.H.; Wang, Y.H.; Chen, C.C. A Subacute Toxicity Study of Cordyceps cicadae Mycelium in High-Glucose Diet-Fed LY Pigs. Hans. J. Food Nutr. Sci. 2016, 5, 13-20.

13. Chen, C.C.; Wong, T.C.; Huang, C.L.; Jhou, B.Y.; Hsu, J.H.; Yeh, S.H.; Tsai, Y.T.; Yang, S.H. Clinical Evaluation of Blood Glucose Regulation and Safety of Cordyceps cicadae Mycelium. J. Food Nutr. Res. 2017, 5, 137-143.

14. Li, I.C.; Hsu, J.H.; Lin, T.W.; Lin, W.H.; Chen, C.C. Prenatal developmental toxicity study of HEA-enriched Cordyceps cicadae mycelia in Sprague-Dawley rats. SDRP J. Food Sci. Technol. 2017, 2, 1-7. [CrossRef]

15. Lin, M.Y.; Hsu, J.H.; Wu, J.J.; Shih, C.H.; Chen, C.C. A Acute Toxicity Study of Cordyceps cicadae Mycelium in ICR Mice and SD Rats. Hans J. Food Nutr. Sci. 2017, 6, 96-105. [CrossRef]

16. Tsai, Y.S.; Hsu, J.H.; David Lin, P.C.; Chang, H.H.; Chang, W.J.; Chen, Y.L.; Chen, C.C. Safety Assessment of HEA-Enriched Cordyceps cicadae Mycelium: A Randomized Clinical Trial. J. Am. Coll. Nutr. 2021, 40, 127-132. [CrossRef]

17. Hsu, J.H.; Chen, C.C. Intraocular Pressure-Lowering Efficacy and Safety of Fermented Cordyceps cicadae Mycelia. Hans J. Food Nutr. Sci. 2021, 10, 33-39.

18. Horng, C.T.; Yang, Y.L.; Chen, C.C.; Huang, Y.S.; Chen, C.; Chen, F.A. Intraocular pressure-lowering effect of Cordyceps cicadae mycelia extract in a glaucoma rat model. Int. J. Med. Sci. 2021, 18, 1007-1014. [CrossRef] [PubMed]

19. Fu, H.I.; Hsu, J.H.; Li, T.J.; Yeh, S.H.; Chen, C.C. Safety assessment of HEA-enriched Cordyceps cicadae mycelia on the central nervous system (CNS), cardiovascular system, and respiratory system in ICR male mice. Food Sci. Nutr. 2021, 9, $4905-4915$. [CrossRef] [PubMed]

20. Lin, T.U.; Chang, H.H.; Tang, U.J.; Chen, C.C.; Lee, Y.L.; David Lin, P.C. Fermented Cordyceps cicadae Mycelia Extracts Ameliorate Dry Eye Symptoms through Reduction of Cornea Epithelial Cell Apoptosis and Maintenance of Conjunctival Goblet Cells in a Mouse Dry Eye Model. Food Nutr. Res. 2017, 5, 320-330. [CrossRef]

21. Fernández-Albarral, J.A.; Ramírez, A.I.; De Hoz, R.; López-Villarín, N.; Salobrar-García, E.; López-Cuenca, I.; Licastro, E.; Inarejos-García, A.M.; Almodóvar, P.; Pinazo-Durán, M.D.; et al. Neuroprotective and Anti-Inflammatory Effects of a Hydrophilic Saffron Extract in a Model of Glaucoma. Int. J. Mol. Sci. 2019, 22, 4110. [CrossRef] [PubMed]

22. Bonomi, L.; Tomazzoli, L.; Jaria, D. An improved model of experimentally induced ocular hypertension in the rabbit. Investig. Ophthalmol. Vis. Sci. 1976, 15, 781-784.

23. Tsai, R.K.; Chang, C.H.; Wang, H.Z. Neuroprotective effects of recombinant human granulocyte colony-stimulating factor (G-CSF) in neurodegeneration after optic nerve crush in rats. Exp. Eye Res. 2008, 87, 242-250. [CrossRef] [PubMed]

24. Albrecht-Küpper, B.E.; Leineweber, K.; Nell, P.G. Partial adenosine A1 receptor agonists for cardiovascular therapies. Purinergic Signal 2012, 8, 91-99. [CrossRef]

25. Varma, R.; Lee, P.; Glodberg, I.; Kotaks, S. An assessment of the health and economic burdens of glaucoma. Am. J. Opthalmol. 2011, 152, 515-522. [CrossRef]

26. Kish, T. Old and New Drug Classes Expanding To Include Glaucoma Treatments. Pharm. Ther. 2018, 43, 489-502. 
27. Tham, Y.C.; Li, X.; Wong, T.Y.; Quigley, H.A.; Aung, T.; Cheng, C.Y. Global prevalence of glaucoma and projections ofglaucoma burden through 2040: A systematic review and meta-analysis. Ophthalmology 2014, 121, 2081-2089. [CrossRef]

28. Bradley, J.M.; Vranka, J.; Colvis, C.M.; Conger, D.M.; Alexander, J.P.; Fisk, A.S.; Samples, J.R.; Acott, T.S. Effect of matrix metalloproteinase activity on outflow in perfused human organ culture. Investig. Ophthalmol. Vis. Sci. 1998, 39, $2649-2658$.

29. Tian, B.; Geiger, B.; Epstein, D.-L.; Kaufman, P.-L. Cytoskeletal involvement in the regulation of aqueous humor outflow. Investig. Ophthalmol. Vis. Sci. 2000, 41, 619-623.

30. Tian, B.; Gabelt, B.T.; Geiger, B.; Kaufman, P.L. The role of the actomyosin system in regulating trabecular fluid outflow. Exp. Eye Res. 2009, 88, 713-717. [CrossRef] [PubMed]

31. Zhong, Y.; Yang, Z.; Huang, W.C.; Luo, X. Adenosine, adenosine receptors and glaucoma: An updated overview. Biochim. Biophys. Acta (BBA)-Gen. Subj. 2013, 1830, 2882-2890. [CrossRef]

32. Jacobson, K.-A.; Tosh, D.K.; Jain, S.; Gao, Z.G. Historical and current adenosine receptor agonists in preclinical and clinical development. Front. Cell. Neurosci. 2019, 13, 124. [CrossRef] [PubMed]

33. Shearer, T.-W.; Crosson, C.E. Adenosine A1 receptor modulation of MMP-2 secretion by trabecular meshwork cells. Investig. Ophthalmol. Vis. Sci. 2002, 43, 3016-3020.

34. Shearer, T.; Crosson, C.E. Activation of extracellular signal-regulated kinase in trabecular meshwork cells. Exp. Eye Res. 2001, 73, 7325-7335. [CrossRef] [PubMed]

35. Li, A.; Leung, C.T.; Peterson-Yantorno, K.; Stamer, W.D.; Mitchell, C.H.; Civan, M.M. Mechanisms of ATP release by human trabecular meshwork cells, the enabling step in purinergic regulation of aqueous humor outflow. J. Cell. Physiol. 2012, 227, 172-182. [CrossRef] [PubMed]

36. Santiago, A.R.; Madeira, M.H.; Boia, R.; Aires, I.D.; Rodrigues-Neves, A.C.; Santos, P.F. Keep an eye on adenosine: Its role in retinal inflammation. Pharmacol. Ther. 2020, 210, 107513. [CrossRef]

37. Hasko, G.; Linden, J.; Cronstein, B.; Pacher, P.A. Adenosine receptors: Therapeutic aspects for inflammatory and immune diseases. Nat. Rev. Drug Discov. 2008, 7, 759-770. [CrossRef]

38. Fishman, P.; Bar-Yehuda, S.; Liang, B.T.; Jacobson, K.A. Pharmacological and therapeutic effects of A3 adenosine receptor agonists Drug Discov. Today 2012, 17, 359-366. [CrossRef]

39. Crosson, C.E. Adenosine receptor activation modulates intraocular pressure in rabbits. J. Pharm. Exp. Ther. 1995, $273,320-326$.

40. Crosson, C.E. Ocular hypotensive activity of the adenosine agonist (R)-phenylisopropyladenosine in rabbits. Curr. Eye Res. 1992, 11, 453-458. [CrossRef]

41. Polska, E.; Ehrlich, P.; Luksch, A.; Fuchsjager-Mayrl, G.; Schmetterer, L. Effects of adenosine on intraocular pressure, optic nerve head blood flow, and choroidal blood flow in healthy humans. Investig. Ophthalmol. Vis. Sci. 2003, 44, 3110-3114. [CrossRef] [PubMed]

42. Ming, F.; Chai, Y.; Chen, G.; Wang, H.; Huang, B. N6-(2-Hydroxyethyl)-adenosine exhibits Insecticidal activity against patella xylostella via Adenosine receptors. PLoS ONE 2016, 11, e0162859.

43. Peng, X.X.; Chai, Y.Q.; Zhu, B.C.; Jin, Y.W.; Li, X.L.; Yu, L.S. The protective effects of N6-(2-hydroxyethyl)-adenosine extracted from Ophiocordyceps sobolifera on renal ischemia reperfusion injury (IRI) in mice. Mycosystema 2015, 34, 11-320.

44. Hu, H.; Lu, W.; Laties, A.M.; Mitchell, C.H. Stimulation of the p2x7 receptor kills rat retinal ganglion cells in vivo. Exp. Eye Res. 2010, 91, 425-432. [CrossRef] 